\title{
Digestive and metabolic effects of potato and maize fibres in human subjects
}

\author{
BY C. CHERBUT ${ }^{1}$, A.-C. AUBE ${ }^{1}$, N. MEKKI ${ }^{2}$, C. DUBOIS ${ }^{2}$, D. LAIRON ${ }^{2}$ \\ AND J.-L. BARRY ${ }^{1}$ \\ ${ }^{1}$ Human Nutrition Research Centre, INRA, BP 71627, 44316 Nantes cedex 03, France \\ ${ }^{2}$ INSERM U-130, 9 avenue Mozart, 13009 Marseilles, France
}

(Received 2 October 1995 - Revised 8 May 1996-Accepted 12 June 1996)

\begin{abstract}
The physiological effects of dietary fibres in humans are due to their physico-chemical properties. However, it is difficult to predict these effects simply by measuring certain characteristics in vitro. Studies in human subjects are still required to assess the effectiveness of new substrates. The aim of the present study in healthy human subjects was to evaluate the effects of two novel fibres, potato (PF) and maize (MF), on fasting and postprandial blood concentrations of carbohydrate and lipid metabolites as well as on stool ouput and transit time. The chemical composition, water-binding capacity (WBC) and fermentative properties of the fibres were also characterized in order to determine their possible involvement in digestive and metabolic effects. Stools, as well as breath and blood samples, were collected after consumption for 1 month of either a basal diet (control) or a basal diet supplemented with fibre (15 g/d). MF resisted fermentation better than PF and had lower digestibility. However, both fibres increased faecal output of dry matter, neutral sugars and water. There was an inverse relationship between stool weight and orofaecal transit time, although only MF significantly reduced transit time. Orocaecal transit was lengthened by PF, probably because of its high WBC. PF ingestion also decreased postprandial plasma levels of total and esterified cholesterol but had no effect on fasting concentrations. In contrast, MF lowered fasting cholesterolaemia and increased free:esterified cholesterol. These particular physiological and fermentative properties suggest that PF and MF would be suitable ingredients in a healthy diet.
\end{abstract}

Dietary fibre: Stool output: Transit time: Plasma lipids

The mechanisms by which a particular dietary fibre acts on human physiology are mainly related to its physico-chemical properties. For instance, solubility seems to be particularly involved in the metabolic effects of fibre. Soluble fibres can apparently reduce serum cholesterol concentration, whereas insoluble ones are usually ineffective (Glore et al. 1994). Other properties, such as viscosity and the ability to sequester bile salts, can also influence glucose and cholesterol metabolism (Wolever, 1995). The effects of dietary fibre on digestive functions also depend on several physico-chemical properties of substrates. It is now recognized that fibre can affect stool weight and transit time through two main mechanisms. First, it may act by increasing lumen volume. This mechanism depends on the amount of indigestible residue in the colon, the water-binding capacity (WBC) of the residue, the stimulation of microbial growth, and the production of gas (Cummings, 1986). The resulting increase in stool volume may cause colonic propulsion through excitation of enteric mechanoreceptors, thus decreasing transit time. Second, fibre may act directly on colonic motility either by mechanical stimulation of submucosal neural receptors (Cherbut \& Ruckebusch, 1985) or by an effect of fermentation end-products, e.g. short-chain fatty acids (SCFA), on the contractile activity of the colon (Cherbut, 1995). 
Even though our understanding of the mechanisms of dietary fibre action is improving, it is still difficult to predict the observable physiological effects of a new fibre preparation in man simply by measuring its physico-chemical and fermentative properties in vitro. Examination of some blood variables and gastrointestinal factors is required to assess the potential action of a novel substrate. The present study in healthy human subjects constitutes the first attempt to evaluate the effects of potato fibre (PF) on blood glucose, insulin, cholesterol and triacylglycerol concentrations and to assess faecal output and composition as well as transit time. The chemical composition, water-holding properties and fermentative pattern of this new fibre are also characterized. In addition, the effects of maize fibre (MF) are studied and compared with those of PF.

\section{MATERIALS AND METHODS}

\section{Characterization of fibres}

Two different dietary fibres were prepared from maize cob and potato pulp (Roquette, Lestrem, France) by mechanical processes (grinding, air-classification, sieving and grinding) and reduced to the same average particle size $(0.08$ (SE 0.02) $\mathrm{mm})$. The chemical composition of the fibre preparations is given in Table 1 . Native substrates were prepared by amylase (EC 3.2.1.1; 3.2.1.3) digestion to remove starch (Prosky et al. 1988). The neutral and acidic sugars of the fibres were determined respectively by GLC (Hoebler et al. 1989) and colorimetry (Thibault, 1979) after the fibres were hydrolyzed in $\mathrm{H}_{2} \mathrm{SO}_{4}$. Each value is given as a proportion of dry matter determined at $103^{\circ}$.

Swelling was measured by the bed-volume technique (Kuniak \& Marchessault, 1972), with slight modification. Dry fibre $(100 \mathrm{mg})$ was weighed into a glass cylinder and left overnight at $25^{\circ}$ in $0.15 \mathrm{M}-\mathrm{NaCl}(\mathrm{pH} 6-6.5)^{?}$. Results are expressed as ml swollen sample/g dry initial sample. WBC was determined by centrifugation (McConnell et al. 1974). Samples $(100 \mathrm{mg})$ were stirred overnight at $25^{\circ}$ in $0.15 \mathrm{M}-\mathrm{NaCl}$ and centrifuged for $1 \mathrm{~h}$ at $14000 \mathrm{~g}$ at $25^{\circ}$. The supernatant fractions were then discarded and the residues left for $1 \mathrm{~h}$ on $\mathrm{G} 2$ sintered glass (porosity $41-100 \mu \mathrm{m}$ ). The pellets were weighed, dried and weighed again. Results are expressed as $\mathrm{g}$ water/g dry residue.

\section{In vitro fermentation}

Fibres were incubated at $40^{\circ}$ in a $\mathrm{N}_{2}$ atmosphere with a human faecal inoculum provided by three healthy subjects accustomed to eating an unspecified Western diet, as previously detailed (Salvador et al. 1993). Three experiments were performed. For each experiment, two batches of each material were prepared, as well as two control batches containing only the faecal inoculum. Fermentation was stopped after 6,12 and $24 \mathrm{~h}$ incubation. Medium $\mathrm{pH}$ was immediately measured in batches removed for analysis. Bottle contents were then

Table 1. Chemical composition of fibre preparations ( $\mathrm{g} / \mathrm{kg} d \mathrm{ry}$ weight)

\begin{tabular}{lcc}
\hline \hline & Maize & Potato \\
\hline Starch & 173 & 237 \\
Protein $(\mathrm{N} \times 6.25)$ & 97.5 & 30 \\
Fat & 23 & 13 \\
Ash & 5 & 18.5 \\
Total fibre & 701.5 & 701.5 \\
\hline \hline
\end{tabular}


centrifuged and the supernatant fraction analysed for SCFA by GLC (Jouany, 1982). Pellets were analysed for cell-wall sugars as described previously. The fermentability of fibre sugars, i.e. the rate of sugar disappearance, was calculated as follows (Salvador et al. 1993):

fermentability $(\%)=100 \times$ (amount of sugars digested/initial amount of sugars).

\section{Subjects}

Eighteen healthy volunteers (ten women and eight men, aged $24-48$ years) gave their informed written consent to the study protocol which was approved by the local Ethics Committee. None had a history of gastrointestinal disease, use of laxatives or recent treatment with antibiotics.

\section{Experimental design}

The subjects were distributed into two groups, one testing MF and the other PF. The study comprised two 1 -month periods with an interval of $21 \mathrm{~d}$. In randomized, blind, cross-over fashion the subjects of each group were given $22 \mathrm{~g}$ (DM basis) of either maize or potato substrate twice daily (providing about $15 \mathrm{~g}$ fibre/d) during one period, and no fibre supplement (control period during which fibre supplement was replaced by sucrose, $15 \mathrm{~g} / \mathrm{d}$ ) during the other period. The fibres were packaged in tubes which also contained $5 \mathrm{~g}$ of the faecal recovery marker, PEG 4000 . The contents of the tubes were mixed with food and ingested during breakfast and dinner.

During each period the subjects consumed a basal diet consisting of conventional lowfibre foods and providing $6 \mathrm{~g}$ dietary fibre and $1-2 \mathrm{~g}$ resistant starch/d, as determined from food tables (Renaud et al. 1979; Englyst et al. 1992). Great care was taken to ensure that the dietary constituents were the same throughout the two periods. A weekly menu was established and rotated each week. The ingredients for breakfast, lunch and dinner were given to the subjects to be prepared and eaten at home. At the end of each period (day 27), subjects were admitted to the clinical centre and given standardized test meals.

During both periods, after $20 \mathrm{~d}$ equilibration, stools were collected from day 21 to day 27 and immediately frozen at $-20^{\circ}$. On days 22,23 and 24 the subjects ingested twenty radiopaque pellets of different shapes with their breakfast to allow measurement of mean orofaecal transit time according to the method of Cummings \& Wiggins (1976). On day 27 a test meal, composed of egg (1), yoghurt (1), cheese $(40 \mathrm{~g})$, vegetable margarine $(20 \mathrm{~g})$, white bread $(60 \mathrm{~g})$, sucrose $(10 \mathrm{~g})$, orange juice $(100 \mathrm{ml})$ and tea or coffee, was given with either $7.5 \mathrm{~g}$ fibre or placebo $(7.5 \mathrm{~g}$ cellulose, Arbocel type B00, Durieux, France). In addition, subjects ingested $10 \mathrm{~g}$ lactulose (Duphalac ${ }^{\circledR}$, Duphar, Villeurbanne, France). End-expiratory breath samples were taken before the meal and every $30 \mathrm{~min}$ thereafter to measure orocaecal transit time over $6 \mathrm{~h}$. Blood samples were taken $15 \mathrm{~min}$ before and immediately before the meal was ingested, and at $30 \mathrm{~min}$ intervals thereafter for $6 \mathrm{~h}$. After test-meal ingestion no food was permitted until the end of exhaled breath gas and blood collection, and subjects had to refrain from smoking.

\section{Analytical methods and calculation}

Each stool was weighed and a portion was lyophilized to measure the dry weight. The $5 \mathrm{~d}$ faecal collections obtained from each subject throughout each period were then pooled. Neutral sugars were measured in each pool as described above. PEG 4000 was measured by the turbidimetric method (Hyden, 1955). 
Concentration of breath $\mathrm{H}_{2}$ was measured using a Quintron model DP instrument (Quintron Microlyser, MI, USA). Orocaecal transit time was defined as the elapsed time between the beginning of the meal and the sustained increase in breath $\mathrm{H}_{2}$ (>15 ppm).

Plasma was separated from whole blood by centrifugation $\left(10^{\circ}, 10 \mathrm{~min}, 910 \mathrm{~g}\right)$. Plasma glucose was measured using the glucose oxidase (EC 1.1.3.4) method (Beckman Analyzer, Fullerton, CA, USA), and plasma insulin was determined by radioimmunoassay (Oris, Gif sur Yvette, France). Triacylglycerols were determined by an enzymic procedure (Buccolo \& David, 1973) using commercial kits (BioMerieux, Marcy l'Etoile, France). Total and free cholesterol were assayed by the cholesterol oxidase (EC 1.1.3.6) method (Siedel et al. 1983) using BioMerieux kits. Phospholipids were assayed by an enzymic procedure (Takayama et al. 1977) using BioMerieux kits. Total serum apoprotein A1 (SievetDesrumeaux et al. 1980) and apoprotein B (Sievet-Desrumeaux et al. 1979) were assayed by laser-nephelometry (Behring Werke AG, Marburg, Germany).

\section{Statistical analysis of data}

Data are expressed as mean and standard error of the mean (SE). Comparisons between the two treatments were made using Wilcoxon's non-parametric test for paired data; $P<0.05$ was considered as the significance level. A two-way ANOVA was performed to test the interaction between treatment (control $v$. fibre) and period (order of distribution). The $P$ value for treatment $\times$ period was above 0.3 for all the considered variables (stool weights, transit time, blood glucose, insulin and lipids) indicating that the wash-out period was

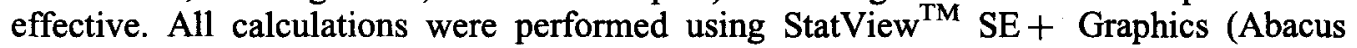
Concepts, Inc., CA, USA) run on a Macintosh computer.

\section{RESULTS}

\section{Fibre characteristics}

The chemical, physico-chemical and fermentative properties of PF and MF were very different. PF contained $140 \mathrm{~g}$ soluble fibre $/ \mathrm{kg}$ and was composed of both neutral (glucose and galactose) and acidic sugars (Table 2), whereas MF was completely insoluble and composed mainly of neutral sugars (arabinose, xylose and glucose). The difference between the total fibre content and soluble plus insoluble fibres of the preparation was about $20 \mathrm{~g} / \mathrm{kg}$, which represents the normal variability between the two methods (Prosky et al. 1988). A totally different method (non-gravimetric, hydrolysis then chromatography or colorimetry) was used to analyse the sugar content of the fibre preparation. This may explain the slight discrepancy between the sum of the neutral and acidic sugars and the total fibre content (Tables 1 and 2). Moreover, it is likely that the analysis of sugars did not take into account all the components of the fibre preparation.

In vitro fermentation of the fibres led to extensive disappearance of $P F$ and low degradation of MF (Table 3). The resulting SCFA profiles also differed, acetatepropionate-butyrate being 66:23:11 and 52:31:17 respectively for PF and MF (Table 3). Although native PF had higher swelling and WBC than MF, it lost this property after in vitro fermentation (Fig. 1).

\section{Faecal outputs}

The percentages of ingested PEG 4000 recovered in stools were 100 (SE 9), 90 (SE 1) and 96 (SE 8) for the control, PF and MF periods respectively. The number of stools was 
Table 2. Chemical composition of maize and potato fibres ( $\mathrm{g} / \mathrm{kg}$ dry weight), determined after starch was removed from the native fibre preparations by amylase digestion*

\begin{tabular}{lrr}
\hline \hline & Maize & Potato \\
\hline Fibre content & & \\
Insoluble & 684 & 588 \\
Soluble & 0 & 96 \\
Neutral sugars & & 8 \\
Rhamnose & 4 & 32 \\
Arabinose & 144 & 14 \\
Xylose & 229 & 8 \\
Mannose & 11 & 145 \\
Galactose & 37 & 206 \\
Glucose & 202 & 197 \\
Acidic sugars & 31 & \\
\hline \hline
\end{tabular}

*For details of procedures, see p. 34.

Table 3. Percentage disappearance (fermentability) and concentration of individual shortchain fatty acids (SCFA) during in vitro incubation of maize and potato fibres* with a human faecal inoculum $\dagger$

(Mean values with their standard errors of three measurements)

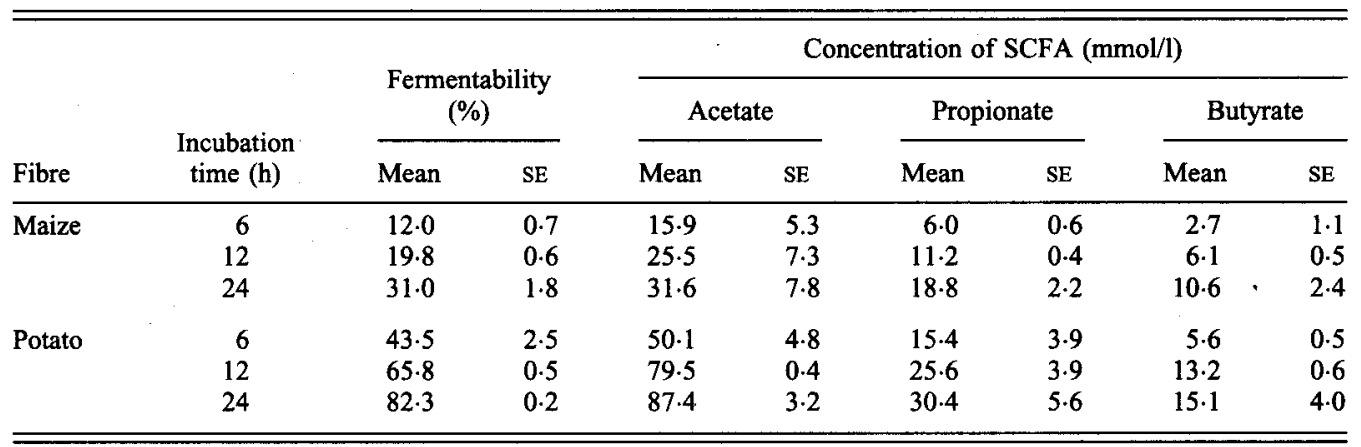

*Before incubation, maize and potato fibre materials were prepared by amylase digestion to remove starch.

†For details of procedures, see pp. 34-35.

slightly increased by ingestion of the fibre preparations (Table 4). Faecal wet and dry weights, as well as faecal water, were significantly greater during the PF and MF periods (Table 4). Accordingly, faecal outputs of neutral sugars increased during MF and PF ingestion (Table 5). However, PF fibre was more degradable than MF throughout the gut: faecal digestibilities of the total neutral sugars of PF and MF, as calculated after correction for the excretion of these sugars during the control period, were respectively 67.9 (SE 12.5; range $19-96$ ) $\%$ and 48.4 (SE 4 ; range $33-66$ ) $\%$.

\section{Transit times}

The increase in breath $\mathrm{H}_{2}$ excretion in each subject after both test meals enabled us to determine orocaecal transit time. Mean orocaecal transit time was not significantly affected by MF consumption but was increased by PF $(P=0.045$, Fig. 2). Mean orofaecal transit 

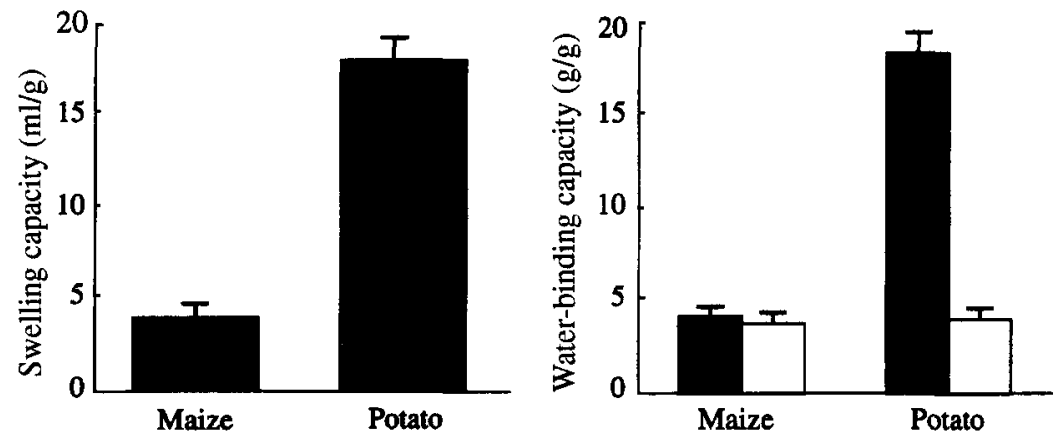

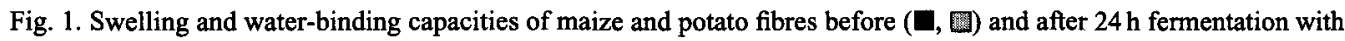
a human faecal inoculum in vitro $(\square)$. For details of procedures see pp. 34-35. Values are means with their standard errors of three measurements.

Table 4. Number of stools and faecal weights recorded in nine subjects for $7 d$ after $20 \mathrm{~d}$ of equilibration to either the control diet (no fibre supplement) or the fibre diet (15 $\mathrm{g}$ maize or potato fibre/d)*

(Mean values with their standard errors; ranges are given in parentheses)

\begin{tabular}{|c|c|c|c|c|c|c|c|c|c|c|c|}
\hline & & \multicolumn{2}{|c|}{$\begin{array}{l}\text { Number of } \\
\text { stools } / \mathrm{d}\end{array}$} & \multicolumn{2}{|c|}{$\begin{array}{c}\text { Faecal wet } \\
\text { weight }(\mathrm{g} / \mathrm{d})\end{array}$} & \multicolumn{2}{|c|}{$\begin{array}{c}\text { Faecal dry } \\
\text { weight }(\mathrm{g} / \mathrm{d})\end{array}$} & \multicolumn{2}{|c|}{$\begin{array}{l}\text { Faecal water } \\
(\mathrm{g} / \mathrm{d})\end{array}$} & \multicolumn{2}{|c|}{$\begin{array}{l}\text { Faecal water } \\
\text { content }(\mathrm{g} / \mathrm{kg})\end{array}$} \\
\hline & & Mean & SE & Mean & SE & Mean & SE & Mean & $\mathrm{SE}$ & Mean & $\mathrm{SE}$ \\
\hline \multirow[t]{2}{*}{ Maize } & $\begin{array}{l}\text { Control } \\
\text { (range) }\end{array}$ & \multicolumn{2}{|c|}{$(0.7-1.0)$} & \multicolumn{2}{|c|}{ (23-93) } & \multicolumn{2}{|c|}{$(7-25)$} & \multicolumn{2}{|c|}{$(16-70)$} & \multicolumn{2}{|c|}{$(670-770)$} \\
\hline & $\begin{array}{l}\text { Fibre } \\
\text { (range) }\end{array}$ & $\begin{array}{l}0.96 \\
(0.7\end{array}$ & $\begin{array}{l}0.06 \\
.2)\end{array}$ & $\begin{array}{r}108.4 \\
(89\end{array}$ & & $\begin{array}{l}32 \cdot 4 \\
\quad(28\end{array}$ & )$^{1.5}$ & $\begin{array}{r}76.9 \\
(61\end{array}$ & 2) & $\begin{array}{l}708 \\
(68\end{array}$ & 7 \\
\hline & & \multicolumn{2}{|c|}{$\begin{array}{c}(0.7-1.2) \\
0.118\end{array}$} & \multicolumn{2}{|c|}{$\begin{array}{c}(89-142) \\
0.007\end{array}$} & \multicolumn{2}{|c|}{$\begin{array}{c}(28-39) \\
0.007\end{array}$} & \multicolumn{2}{|c|}{$\begin{array}{c}(61-102) \\
0.007\end{array}$} & & \\
\hline \multirow{2}{*}{ Potato } & $\begin{array}{l}\text { Control } \\
\text { (range) }\end{array}$ & \multicolumn{2}{|c|}{$(0 \cdot 7-1 \cdot 1)$} & \multicolumn{2}{|c|}{$(44-115)$} & \multicolumn{2}{|c|}{$(11-31)$} & \multicolumn{2}{|c|}{$(33-92)$} & \multicolumn{2}{|c|}{$(710-820)$} \\
\hline & $\begin{array}{l}\text { Fibre } \\
\text { (range) }\end{array}$ & $\begin{array}{l}1.1 \\
(0.6\end{array}$ & $\begin{array}{l}0.14 \\
.5)\end{array}$ & $\begin{array}{r}115 \cdot 7 \\
\quad(59\end{array}$ & $\begin{array}{l}9 \cdot 1 \\
1)\end{array}$ & $\begin{array}{l}28 \cdot 3 \\
(17\end{array}$ & 8) & $\begin{array}{l}86.9 \\
\quad(42\end{array}$ & $7 \cdot 5$ & $\begin{array}{l}748 \\
\quad(70\end{array}$ & 13 \\
\hline$P$ value & & \multicolumn{2}{|c|}{0.088} & \multicolumn{2}{|c|}{0.011} & \multicolumn{2}{|c|}{0.011} & \multicolumn{2}{|c|}{0.011} & \multicolumn{2}{|c|}{0.262} \\
\hline
\end{tabular}

*The experimental periods (control and fibre) were separated by an interval of $21 \mathrm{~d}$. For details of diets, see p. 35.

time was not changed during the PF period but was significantly reduced during the MF period $(P=0.011$, Fig. 2$)$. By combining the data for each subject during all periods $(n 36)$ we calculated a significant inverse relationship between orofaecal transit time and faecal wet weight $(y=-0.42 x+114, r 0.530, P=0.001)$.

\section{Blood variables}

Fasting plasma concentrations of glucose, insulin and triacylglycerols were not affected by consumption for 1 month of either MF or PF (Table 6). However, MF ingestion 
Table 5. Faecal outputs of neutral sugars $(\mathrm{g} / \mathrm{d})$ recorded in nine subjects after $20 \mathrm{~d}$ of equilibration to either the control diet (no fibre supplement) or the fibre diet (15 $\mathrm{g}$ maize or potato fibre/d)*

(Mean values with their standard errors; ranges are given in parentheses)

\begin{tabular}{|c|c|c|c|c|c|c|c|c|c|c|}
\hline \multirow[b]{3}{*}{ Sugars } & \multicolumn{4}{|c|}{ Maize } & \multirow[b]{3}{*}{$P$ value } & \multicolumn{4}{|c|}{ Potato } & \multirow[b]{3}{*}{$P$ value } \\
\hline & \multicolumn{2}{|c|}{ Control } & \multicolumn{2}{|c|}{ Fibre } & & \multicolumn{2}{|c|}{ Control } & \multicolumn{2}{|c|}{ Fibre } & \\
\hline & Mean & SE & Mean & SE & & Mean & SE & Mean & $\mathrm{SE}$ & \\
\hline $\begin{array}{l}\text { Rhamnose } \\
\text { (range) }\end{array}$ & $\begin{array}{c}0.13 \\
(0.0\end{array}$ & $\begin{array}{l}0.01 \\
19)\end{array}$ & $\begin{array}{r}0.28 \\
(0.1\end{array}$ & $\begin{array}{l}0.09 \\
.0)\end{array}$ & 0.015 & $\begin{array}{l}0.14 \\
\quad(0.0\end{array}$ & $\begin{array}{l}0.03 \\
29)\end{array}$ & $\begin{array}{r}0.20 \\
(0.1\end{array}$ & $\begin{array}{l}0.03 \\
.38)\end{array}$ & 0.017 \\
\hline $\begin{array}{l}\text { Arabinose } \\
\text { (range) }\end{array}$ & $\begin{array}{r}0.22 \\
(0-\end{array}$ & $\begin{array}{l}0.08 \\
2)\end{array}$ & $\begin{array}{l}1.61 \\
(1 \cdot 1\end{array}$ & $\begin{array}{l}0 \cdot 13 \\
.40)\end{array}$ & 0.007 & $\begin{array}{l}0.17 \\
\quad(0.0\end{array}$ & $\begin{array}{l}0.03 \\
32)\end{array}$ & $\begin{array}{l}0.30 \\
(0.0\end{array}$ & $\begin{array}{l}0.06 \\
.65)\end{array}$ & 0.012 \\
\hline $\begin{array}{l}\text { Xylose } \\
\text { (range) }\end{array}$ & $\begin{array}{r}0.25 \\
(0\end{array}$ & $\begin{array}{l}0.07 \\
8)\end{array}$ & $\begin{array}{l}2.51 \\
(1.6\end{array}$ & $\begin{array}{l}0.20 \\
-53)\end{array}$ & 0.007 & $\begin{array}{l}0.25 \\
(0.0\end{array}$ & $\begin{array}{l}0.06 \\
52)\end{array}$ & $\begin{array}{r}0.47 \\
(0.1\end{array}$ & $\begin{array}{l}0 \cdot 11 \\
.92)\end{array}$ & 0.017 \\
\hline $\begin{array}{l}\text { Mannose } \\
\text { (range) }\end{array}$ & $\begin{array}{r}0.11 \\
(0\end{array}$ & $\begin{array}{l}0.03 \\
8)\end{array}$ & $\begin{array}{l}0.17 \\
(0.0\end{array}$ & $\begin{array}{l}0.02 \\
26)\end{array}$ & 0.109 & $\begin{array}{l}0.14 \\
\quad(0.0\end{array}$ & $\begin{array}{l}0.03 \\
27)\end{array}$ & $\begin{array}{l}0.34 \\
\quad(0.0\end{array}$ & $\begin{array}{l}0.14 \\
.23)\end{array}$ & 0.093 \\
\hline $\begin{array}{l}\text { Galactose } \\
\text { (range) }\end{array}$ & $\begin{array}{l}0.25 \\
(0.1\end{array}$ & $\begin{array}{l}0.02 \\
34)\end{array}$ & $\begin{array}{l}0.75 \\
(0.5\end{array}$ & $\begin{array}{l}0.05 \\
.04)\end{array}$ & 0.007 & $\begin{array}{c}0.27 \\
(0.1\end{array}$ & $\begin{array}{l}0.04 \\
40)\end{array}$ & $\begin{array}{l}0.50 \\
(0.1\end{array}$ & $\begin{array}{c}0.11 \\
.02)\end{array}$ & 0.025 \\
\hline $\begin{array}{l}\text { Glucose } \\
\text { (range) }\end{array}$ & $\begin{array}{c}0.88 \\
(0.2\end{array}$ & $\begin{array}{l}0 \cdot 17 \\
81)\end{array}$ & $\begin{array}{l}3 \cdot 17 \\
(2 \cdot 0\end{array}$ & $\begin{array}{l}0.26 \\
.61)\end{array}$ & 0.007 & $\begin{array}{c}1.53 \\
(0.3\end{array}$ & $\begin{array}{l}0.61 \\
95)\end{array}$ & $\begin{array}{l}3.56 \\
(0.72\end{array}$ & $\begin{array}{l}1.39 \\
.22)\end{array}$ & 0.012 \\
\hline $\begin{array}{l}\text { Total } \\
\text { (range) }\end{array}$ & $\begin{array}{r}1.84 \\
(0.6\end{array}$ & $\begin{array}{l}0.34 \\
10)\end{array}$ & $\begin{array}{l}8.54 \\
(5.90\end{array}$ & $\begin{array}{l}0.62 \\
.97)\end{array}$ & 0.007 & $\begin{array}{r}2.49 \\
(0.7\end{array}$ & $\begin{array}{l}0.77 \\
50)\end{array}$ & $\begin{array}{l}5.38 \\
(1.29\end{array}$ & $\begin{array}{l}1.77 \\
3.38)\end{array}$ & 0.012 \\
\hline
\end{tabular}

*The experimental periods (control and fibre) were separated by an interval of $21 \mathrm{~d}$. For details of diets, see p. 35 .

significantly reduced the basal concentrations of total and esterified cholesterol $(P=0.045$ and 0.038 respectively), and increased the free:esterified cholesterol ratio $(P=0.065)$. $\mathrm{PF}$ had no significant effect (Table 6).

Postprandial plasma levels of glucose, insulin and triacylglycerols were not changed by MF and PF (Fig. 3). Although MF ingestion did not modify the postprandial areas under the curve (AUC) for total, esterified and free cholesterol, PF consumption caused a significant reduction in AUC for total and esterified cholesterol $(P=0.038$ and 0.019 respectively, Table 7 ).

As sucrose $(15 \mathrm{~g} / \mathrm{d})$ was used as a placebo, we cannot exclude the possibility that it may have affected plasma glucose, insulin and lipid concentrations. However, $15 \mathrm{~g}$ sucrose/d provides only $2.7 \%$ energy on the basis of a $10.46 \mathrm{MJ}$ daily intake. Thus, it is unlikely that this low consumption of sucrose as a percentage of energy would have caused major changes in blood variables. In addition, in normal men and women, $50-107 \mathrm{~g}$ sucrose $/ \mathrm{d}$, for 7-14 d, did not affect fasting glucose and insulin levels in the serum (Bossetti et al. 1984) and significant increases in plasma triacylglycerols and cholesterol concentrations were reported with much higher daily sucrose intakes (Macdonald, 1964; Bagley et al. 1976). 

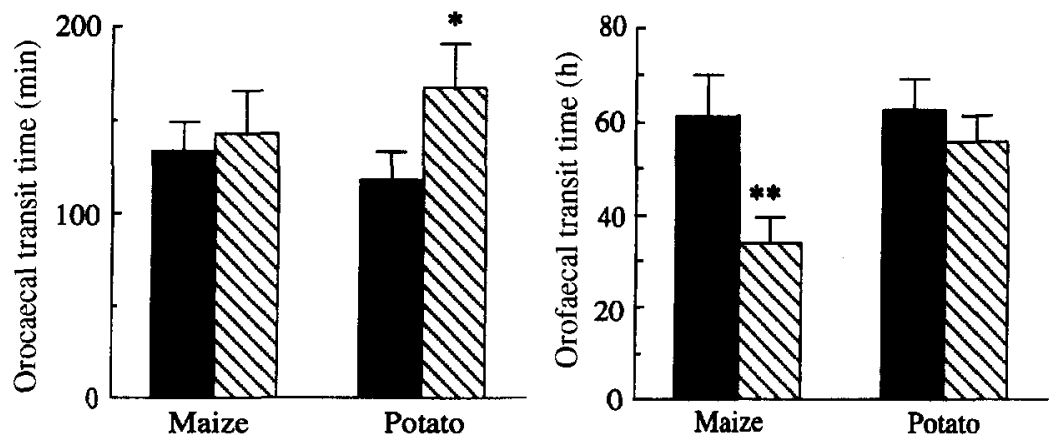

Fig. 2. Orocaecal and orofaecal transit times measured in subjects after 20-27 d of equilibration to either the control diet (no fibre supplement, $\square$ ) or the fibre diet $(15 \mathrm{~g}$ maize or potato fibre $/ \mathrm{d}, \mathbb{N}$ ). Values are means with their standard errors for nine subjects. Mean values were significantly different from control, ${ }^{*} P<0.05,{ }^{* *} P<0.01$.

Table 6. Faecal plasma concentrations of glucose, insulin, lipids and apolipoproteins measured in nine subjects after 4 weeks ingestion of either the control diet (no fibre supplement) or the fibre diet (15 $\mathrm{g}$ maize or potato fibre/d)*

(Mean values with their standard errors; ranges are given in parentheses)

\begin{tabular}{|c|c|c|c|c|c|c|}
\hline & \multicolumn{2}{|c|}{ Maize } & \multirow[b]{3}{*}{$P$ value } & \multicolumn{2}{|c|}{ Potato } & \multirow[b]{3}{*}{$P$ value } \\
\hline & Control & Fibre & & Control & Fibre & \\
\hline & Mean & Mean & & Mean & Mean & \\
\hline $\begin{array}{l}\text { Glucose (mmol/l) } \\
\text { (range) }\end{array}$ & $\begin{array}{cc}4 \cdot 7 & 1 \cdot 2 \\
(3 \cdot 7-5 \cdot 5) & \end{array}$ & $\begin{array}{c}4 \cdot 6 \quad 1 \cdot 2 \\
(3 \cdot 5-5 \cdot 4)\end{array}$ & 0.767 & $\begin{array}{c}4 \cdot 0 \quad 1 \cdot 4 \\
(3 \cdot 1-5 \cdot 2)\end{array}$ & $\begin{array}{c}3 \cdot 9 \quad 1 \cdot 2 \\
(3 \cdot 2-5 \cdot 0)\end{array}$ & 0.523 \\
\hline $\begin{array}{l}\text { Insulin (mU/l) } \\
\text { (range) }\end{array}$ & $\begin{array}{cc}12 \cdot 1 & 1.0 \\
(6.4 & 17 \cdot 5)\end{array}$ & $\begin{array}{cc}11 \cdot 2 & 1 \cdot 3 \\
(5 \cdot 0-17 \cdot 5)\end{array}$ & 0.514 & $\begin{array}{cc}9.7 & 0.9 \\
(6.9 & -13.6)\end{array}$ & $\begin{array}{cc}8 \cdot 2 & 1 \cdot 1 \\
(0-11 \cdot 0)\end{array}$ & 0.310 \\
\hline $\begin{array}{l}\text { Triacylglycerols }(\mathrm{mmol} / \mathrm{l}) \\
\text { (range) }\end{array}$ & $\begin{array}{cc}0.9 & 0.1 \\
(0.5-1.7)\end{array}$ & $\begin{array}{l}0.9 \quad 0.08 \\
(0.5-1.3)\end{array}$ & 0.767 & $\begin{array}{ll}0.9 & 0.04 \\
(0.7-1.4)\end{array}$ & $\begin{array}{c}0.9 \quad 0.1 \\
(0.4-1.7)\end{array}$ & 0.515 \\
\hline $\begin{array}{l}\text { Cholesterol } \\
\text { total }(\mathrm{mmol} / \mathrm{l}) \\
\text { (range) }\end{array}$ & $\begin{array}{ll}5.7 & 0.2 \\
(4.2 & 6 \cdot 6)\end{array}$ & $\begin{array}{cc}5.0 & 0.3 \\
(3.6-6.2)\end{array}$ & 0.045 & $\begin{array}{l}5.8 \quad 0.5 \\
(4.0-7 \cdot 0)\end{array}$ & $\begin{array}{l}5 \cdot 1 \quad 0.3 \\
(3 \cdot 8-6 \cdot 5)\end{array}$ & 0.138 \\
\hline $\begin{array}{l}\text { esterified (mmol/l) } \\
\quad \text { (range) }\end{array}$ & $\begin{array}{cc}4.6 & 0.4 \\
(2 \cdot 6-7.4)\end{array}$ & $\begin{array}{cc}3.6 & 0.3 \\
(2.4-4.7)\end{array}$ & 0.038 & $\begin{array}{cc}4.4 & 0.3 \\
(2 \cdot 9-5 \cdot 5)\end{array}$ & $\begin{array}{l}3.9 \quad 0.3 \\
(2 \cdot 8-5 \cdot 2)\end{array}$ & 0.260 \\
\hline $\begin{array}{l}\text { free }(\mathrm{mmol} / \mathrm{l}) \\
\text { (range) }\end{array}$ & $\begin{array}{cc}1 \cdot 1 & 0 \cdot 1 \\
(0.3-1 \cdot 6)\end{array}$ & $\begin{array}{c}1.4 \quad 0.07 \\
(1.0-1.7)\end{array}$ & 0.314 & $\begin{array}{c}1.3 \quad 0.09 \\
(1 \cdot 1-1 \cdot 8)\end{array}$ & $\begin{array}{c}1.2 \quad 0.08 \\
(0.9-1.6)\end{array}$ & 0.085 \\
\hline $\begin{array}{l}\text { free:esterified } \\
\quad \text { (range) }\end{array}$ & $\begin{array}{cc}0.27 & 0.05 \\
(0.11-0.29)\end{array}$ & $\begin{array}{cc}0.38 & 0.02 \\
(0.29-0.4)\end{array}$ & 0.065 & $\begin{array}{cc}0.31 & 0.01 \\
(0.29-0.33)\end{array}$ & $\begin{array}{cc}0.32 & 0.02 \\
(0.28-0.4)\end{array}$ & 0.138 \\
\hline $\begin{array}{l}\text { Phospholipids (mmol/1) } \\
\text { (range) }\end{array}$ & $\begin{array}{ll}4 \cdot 1 & 0.2 \\
(2 \cdot 7-5 \cdot 1)\end{array}$ & $\begin{array}{ll}2.8 & 0.6 \\
(1.7-7.0)\end{array}$ & 0.109 & $\begin{array}{cc}2.5 & 0.3 \\
(1.7-4.4)\end{array}$ & $\begin{array}{l}2.0 \quad 0.1 \\
(1.4-2 \cdot 5)\end{array}$ & 0.085 \\
\hline $\begin{array}{l}\text { Apolipoprotein A1 (mg/l) } \\
\text { (range) }\end{array}$ & $\begin{array}{ll}1.8 & 0.18 \\
(1.3-2.76)\end{array}$ & $\begin{array}{cc}1.41 & 0.11 \\
(0.98-2.0)\end{array}$ & 0.093 & $\begin{array}{l}1.30 \quad 0.68 \\
(1.0-1.58)\end{array}$ & $\begin{array}{ll}1.26 & 0.68 \\
(0.94-1.5)\end{array}$ & 0.593 \\
\hline $\begin{array}{l}\text { Apolipoprotein B (mg/l) } \\
\text { (range) }\end{array}$ & $\begin{array}{cc}0.93 & 0.73 \\
(0.62-1.28)\end{array}$ & $\begin{array}{lr}0.9 & 0.6 \\
(0.58-1.17)\end{array}$ & 0.674 & $\begin{array}{cc}0.90 & 0.54 \\
(0.75-1.24)\end{array}$ & $\begin{array}{cc}0.87 & 0.39 \\
(0.72-1.08)\end{array}$ & 0.767 \\
\hline
\end{tabular}

*The experimental periods (control and fibre) were separated by an interval of $21 \mathrm{~d}$. For details of diets, see p. 35. 

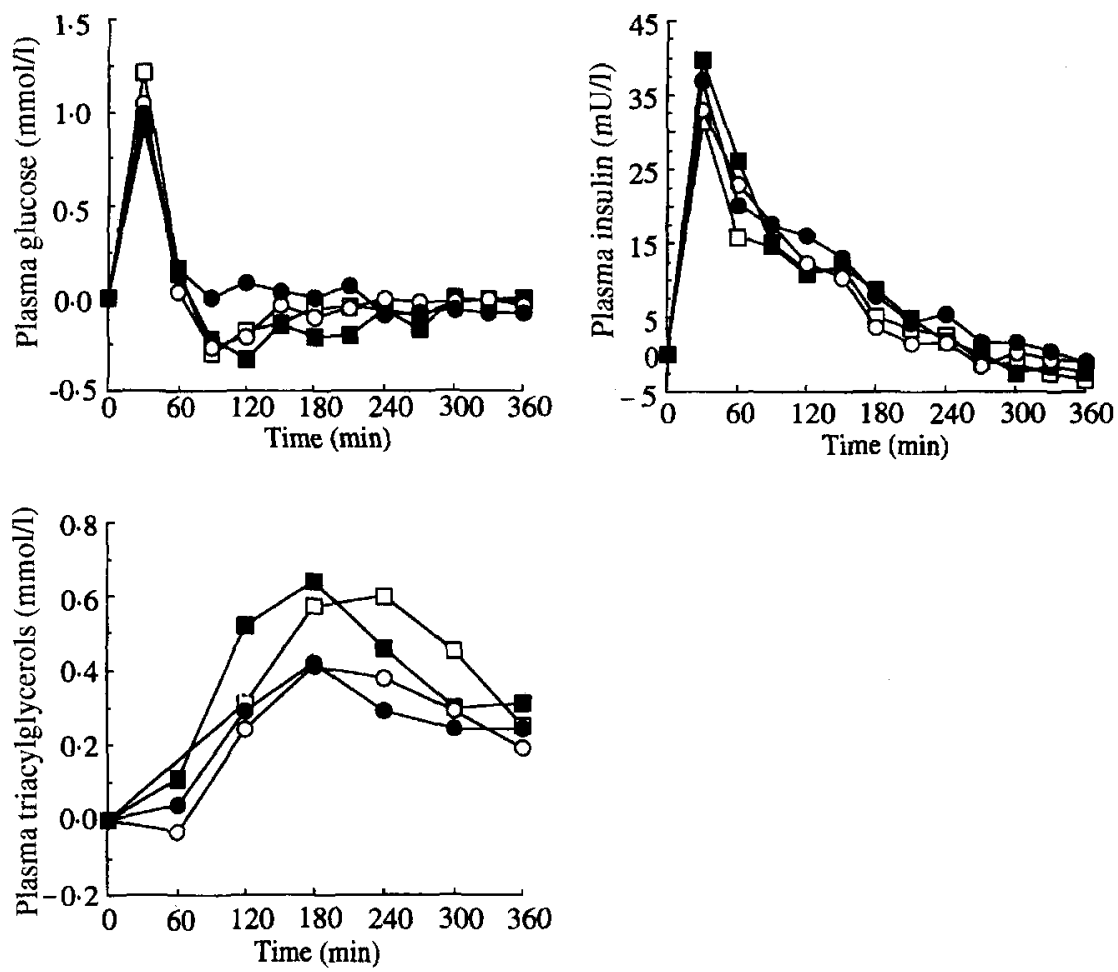

Fig. 3. Incremental plasma glucose, insulin and triacylglycerol concentrations measured after ingestion of a test meal containing either placebo $(7.5 \mathrm{~g}$ cellulose, $\square$ and $O$ ) or maize $(\square)$ or potato $(O)$ fibre $(7.5 \mathrm{~g})$. The response to the test meal was determined after 4 weeks of equilibration to either the control diet (no fibre supplement) or the fibre diet ( $15 \mathrm{~g}$ maize or potato fibre/d). Values are means for nine subjects. Plasma concentrations were not significantly different after placebo and fibres.

\section{DISCUSSION}

$\mathrm{PF}$, a novel substrate rich in soluble fibre, and MF proved to be effective stool bulkers in humans. However, only MF, which was poorly degraded throughout the gut, shortened orofaecal transit time. In addition to providing a bulking effect, PF modified orocaecal transit time and decreased the postprandial plasma concentrations of total and esterified cholesterol. Although MF had no effect on the postprandial level of measured metabolic variables, its consumption for 1 month caused a reduction in the fasting concentrations of cholesterol and phospholipids.

Several mechanisms may be involved in the increase in stool weight caused by MF and PF. Dietary fibre actually affects stool output by increasing unfermented residues, faecal water and microbial mass (Cummings, 1986; Edwards, 1995). In the present study, consumption of MF and PF increased faecal output of neutral sugars, accounting in part for the increase in the dry weight of stools. MF appeared to be more resistant to degradation than PF. Indeed, both in vitro and in vivo, the percentage disappearance of MF was significantly lower than that of PF. This behaviour could be due to the chemical structure of MF as well as to its low WBC. Insoluble fibres are known to be less degraded by colonic flora than soluble ones (Mortensen \& Norgaard-Andersen, 1993). Furthermore, MF is composed mainly of xylose, arabinose and cellulosic glucose. In this respect, MF is close to 
Table 7. Area under the $0-6 \mathrm{~h}$ curves of postprandial incremental concentrations of plasma lipids measured in nine subjects who ingested a test meal together with either $7.5 \mathrm{~g}$ cellulosed (placebo) or $7.5 \mathrm{~g}$ maize or potato fibre*

(Mean values with their standard errors; ranges are given in parentheses)

\begin{tabular}{|c|c|c|c|c|c|c|c|c|}
\hline & \multicolumn{2}{|c|}{ Maize } & \multirow[b]{3}{*}{$P$ value } & \multicolumn{4}{|c|}{ Potato } & \multirow[b]{3}{*}{$P$ value } \\
\hline & Control & Fibre & & \multicolumn{2}{|c|}{ Control } & \multicolumn{2}{|c|}{ Fibre } & \\
\hline & Mean & Mean & & Mean & SE & Mean & $\mathrm{SE}$ & \\
\hline $\begin{array}{l}\text { Triacylglycerols (mmol.h/l) } \\
\text { (range) }\end{array}$ & $\begin{array}{lr}2.65 & 0.27 \\
(2.05,3.21)\end{array}$ & $\begin{array}{ll}2.70 & 0.77 \\
(2 \cdot 10,3 \cdot 49)\end{array}$ & 0.765 & $\begin{array}{l}1.89 \\
(1.54\end{array}$ & $\begin{array}{r}0.39 \\
2.86)\end{array}$ & $\begin{array}{l}1.88 \\
(1.50,2\end{array}$ & $\begin{array}{r}0.22 \\
2.27)\end{array}$ & 0.767 \\
\hline $\begin{array}{l}\text { Total cholesterol (mmol.h/l) } \\
\text { (range) }\end{array}$ & $\begin{array}{cc}-5.97 & 1.89 \\
(-7.09, & -2.84)\end{array}$ & $\begin{array}{ll}-2.96 & 5.09 \\
(-8.87, & 1.53)\end{array}$ & 0.374 & $\begin{array}{l}-0.42 \\
(-2.73\end{array}$ & $\begin{array}{l}1.04 \\
0.96)\end{array}$ & $\begin{array}{l}-2.32 \\
(-3.95\end{array}$ & $\begin{array}{c}1.12 \\
-1.34)\end{array}$ & 0.038 \\
\hline $\begin{array}{l}\text { Esterified cholesterol (mmol.h/l) } \\
\text { (range) }\end{array}$ & $\begin{array}{lc}-5.46 & 0.80 \\
(-6.72, & -3.47)\end{array}$ & $\begin{array}{ll}-2.34 & 1.05 \\
(-5 \cdot 19, & -0.68)\end{array}$ & 0.213 & $\begin{array}{l}0.85 \\
(-1.23\end{array}$ & $\begin{array}{c}1.22 \\
2 \cdot 09)\end{array}$ & $\begin{array}{l}-2.25 \\
(-3.95\end{array}$ & $\begin{array}{l}1.84 \\
0.57)\end{array}$ & 0.019 \\
\hline $\begin{array}{l}\text { Free cholesterol }(\mathrm{mmol} . \mathrm{h} / \mathrm{l}) \\
\quad \text { (range) }\end{array}$ & $\begin{array}{ll}-0.50 & 0.80 \\
(-2 \cdot 14, & 1 \cdot 39)\end{array}$ & $\begin{array}{lc}-0.71 & 0.24 \\
(-1.65, & 0.83)\end{array}$ & $0 \cdot 314$ & $\begin{array}{l}-3 \cdot 81 \\
(-4 \cdot 23\end{array}$ & $\begin{array}{c}2.84 \\
-0.58)\end{array}$ & $\begin{array}{l}0.03 \\
(-0.89\end{array}$ & $\begin{array}{l}0.34 \\
0.42)\end{array}$ & 0.953 \\
\hline $\begin{array}{l}\text { Phospholipids (mmol.h/l) } \\
\text { (range) }\end{array}$ & $\begin{array}{lr}5.24 & 2.63 \\
(2.45,7.11)\end{array}$ & $\begin{array}{lr}1.93 & 2.81 \\
(0.98,5.07)\end{array}$ & 0.441 & $\begin{array}{l}-0.78 \\
(-2.38,\end{array}$ & $\begin{array}{r}0.69 \\
0.95)\end{array}$ & $\begin{array}{l}-0.68 \\
(-1.87,\end{array}$ & $\begin{array}{l}0.46 \\
0.48)\end{array}$ & 0.374 \\
\hline
\end{tabular}

* The postprandial response to the test meal was determined after 4 weeks of equilibration to either the control diet (no fibre supplement) or to the fibre diet ( $15 \mathrm{~g}$ maize or potato fibre/d). For details of procedures, see pp. 35-36.

wheat-bran fibre which is composed largely of arabinoxylans and cellulose. Numerous studies have shown that wheat bran is poorly hydrolysed by colonic bacteria, in part because the highly branched structure of arabinoxylans limits accessibility to bacteria (Brillouet \& Mercier, 1981; Stevens \& Selvendran, 1988). Conversely, PF is rich in extensively fermented uronic acids (Van Soest et al. 1983; Stevens et al. 1988; Salvador et al. 1993) and galactose, a readily degraded sugar (Guillon et al. 1995). In addition, PF had a high WBC, which should make it more metabolizable since a positive relationship has been found between WBC and fibre fermentability (McBurney et al. 1985; Auffret et al. 1993).

About $6 \mathrm{~g} / \mathrm{d}$ of the stool dry weight cannot be accounted for by unfermented neutral sugars. We measured neither $N$ nor lignin content in the stools, nevertheless we may hypothesize that this extra faecal DM could come from endogenous materials (mucins, sloughed epithelial cells, etc.) and bacteria, and possibly from other undegraded fibre components, such as proteins or polyphenols.

The two fibre preparations also increased faecal water output, which may have been due to several causes. First, the increase could have been related to excreted residues. Although native PF had a high WBC, it lost this property after being degraded by colonic flora. Its WBC (less than $5 \mathrm{~g} / \mathrm{g}$ ) was then quite similar to that of MF. Although this WBC can account for all the extra faecal water with $\mathrm{MF}$, it cannot explain the $30 \%$ increase in water output induced by PF. Indeed, about $15 \mathrm{~g}$ stool water $/ \mathrm{d}$ was not bound to the unfermented PF. This extra water might have come from faecal microbes since bacteria contain about $80 \%$ water (Macfarlane \& Cummings, 1991). As PF is extensively metabolized by colonic flora, its ingestion may favour microbial growth, thus increasing the bacterial mass in stools. Finally, the excreted water could have been free water not absorbed by the colon because of a short transit time or changes in colonic motility. It has been shown in dogs (Cherbut \& Ruckebusch, 1985) that ingestion of plastic particles 
inhibits the colonic contractions responsible for the mixing and retention of contents and thus increases stool water output.

As previously reported by Spiller (1986), stool weight was inversely related to orofaecal transit time. However, only MF had a significant effect on total transit time. It is recognized that poorly fermented fibres can reduce colonic retention time through a physical mechanism (Bardon \& Fioramonti, 1983; Cherbut et al. 1991). This effect is due either to an increase in the volume of digesta, inducing distension of the colon which stimulates propulsive motility (Narducci et al. 1987), or to excitation of submucosal mechanoreceptors (Grider \& Jin, 1994) by the edges of fibre particles. Although PF did not alter orofaecal transit time, it lengthened orocaecal transit, an effect possibly related to its high swelling and WBC. It has already been observed that fibres exhibiting a high WBC, such as those of peas or sugarbeet, slow the rate of orocaecal transit (Shinnick et al. 1989; Cherbut et al. 1991). Furthermore, sugarbeet and ispaghula, which have WBC similar to that of PF, modify the motility of the human small intestine (Cherbut et al. 1994).

The mechanisms by which dietary fibre influences cholesterol metabolism are still incompletely elucidated. Nevertheless, there is evidence that fibre may affect cholesterolaemia in at least five ways: altered absorption of fat and cholesterol (Borel et al. 1989), modified postprandial lipaemia and lipoproteins (Cara et al. 1992; Dubois et al. 1993), reduced insulin stimulus to cholesterol synthesis (Wolever, 1995), increased faecal bile acid excretion (Anderson et al. 1990; Jenkins et al. 1993), and systemic effects of the SCFA produced during colonic fibre fermentation (Anderson et al. 1991). The different metabolic effects observed after either PF or MF supplementation suggest that these fibre preparations are not governed by the same mechanisms. PF had no influence on fasting plasma lipid variables after 4 weeks intake but reduced postprandial plasma cholesterol concentration. It has already been reported that some fractions rich in insoluble fibres, such as concentrated wheat bran (Cara et al. 1992) or pea fibre (Dubois et al. 1993), can lower total and esterified cholesterol in plasma postprandially. This effect might be related to an alteration in lipid digestion and absorption in the upper gut (Borel et al. 1989). Actually, $\mathrm{PF}$, which has notable swelling and water-holding capacities, may have modified the physico-chemistry of the intestinal contents, thereby impeding the mixing of food with digestive secretions as well as the convection of nutrients toward the absorptive area. The increase in orocaecal transit time induced by PF may account for this mechanism. Moreover, as previously suggested (Cara et al. 1992; Morgan et al. 1993), a postprandial decrease in plasma cholesterol might originate from an increased demand from the liver for de novo bile salt synthesis during the digestive period.

In contrast to $\mathrm{PF}$, MF did not alter postprandial cholesterolaemia but elicited significant reductions in the total and esterified cholesterol of fasting plasma after 1 month of ingestion. Chronic consumption of MF decreased plasma total cholesterol $(-12.2 \%)$ as well as the free:esterified value. Comparable changes have been reported with soluble fibres such as guar gum, psyllium or oat bran (Jenkins et al. 1979; Anderson et al. 1984; Wolever et al. 1994). However, it is quite unusual for an insoluble fibre to produce such an effect (Krestin et al. 1990; Lampe et al. 1991). It is unlikely that SCFA, especially propionate (Stephen, 1994), could be involved in this effect. Low fibre fermentability was measured in vitro and in vivo, and the production of propionate was relatively low. Concerning the bile acid mechanism, MF may bind bile acids in the intestine and increase their excretion in stools, as has been observed for other cereal fibres (Story \& Kritchevsky, 1976). This effect interrupts enterohepatic circulation, increases hepatic conversion of cholesterol into bile acids and results in lowered blood LDL and total cholesterol (Wolever, 1995). However, we did not measure either the in vitro bile acid-binding capacity of the 
fibre or the faecal bile acid output. The fact that fasting plasma phospholipids and apolipoprotein (apo) A1 decreased simultaneously ( -31.7 and $-21.9 \%$ respectively), but apo B did not, suggests that a drop in HDL particles was the likely cause of the cholesterol changes observed. Further dedicated studies will be necessary for a fuller understanding of the mechanisms by which sources of insoluble fibres such as MF or wheat bran (Kashtan et al. 1992) can alter cholesterol metabolism.

Thus, daily consumption of PF and MF, even in relatively small quantities $(15 \mathrm{~g} / \mathrm{d}$, representing about half of the recommended intake of dietary fibre), produced changes in digestive and metabolic physiology which may be considered beneficial for consumer health. Both fibres increased stool output, and MF also shortened orofaecal transit time. Moreover, both were fermented in the colon and produced SCFA. It has been suggested that all these effects can potentially reduce the risk of colon cancer (Gurr \& Asp, 1994). In addition to their digestive action, PF and MF influenced cholesterol metabolism by reducing postprandial or fasting cholesterolaemia respectively. Furthermore, MF was able to decrease total and esterified cholesterol, an effect which could play a role in reducing the risk of coronary heart disease.

The authors wish to thank A. Davis, M. Rival and C. Bonnet for their technical assistance and Roquette Frères (Lestrem, France) for financial support.

\section{REFERENCES}

Anderson, J. W., Deakins, D. A. \& Bridges, S. R. (1990). Soluble fiber: hypocholesterolemic effects and proposed mechanisms. In Dietary Fiber: Chemistry, Physiology, and Health Effects, pp. $339-363$ [D. Kritchevsky, C. Bonfield and J. W. Anderson, editors]. New York: Plenum Press.

Anderson J. W., Gilinsky, N. H., Deakins, D. A., Smith, S. F., O'Neal, D. S., Dillon, D. W. \& Oeltgen, P. R. (1991). Lipid responses of hypercholesterolemic men to oat-bran and wheat-bran intake. American Journal of Clinical Nutrition 54, 678-686.

Anderson, J. W., Story, L., Sieling, B., Chen, W.-J. L., Petro, M. S. \& Story, J. (1984). Hypocholesterolemic effects of oat bran or bean intake for hypercholesterolemic men. American Journal of Clinical Nutrition 40 , 1146-1155.

Auffret, A., Barry, J. L. \& Thibault, J. F. (1993). Effect of chemical treatments of sugar beet fibre on their physico-chemical properties and on their in vitro fermentation. Journal of the Science of Food and Agriculture 61, 195-203.

Bagley, R., Ford, M. \& Grenn, L. F. (1976). Changes in plasma lipids after substituting glucose-syrup solids for table sucrose. Proceedings of the Nutrition Society 35, 68A-69A.

Bardon, T. \& Fioramonti, J. (1983). Nature of the effects of bran on digestive transit time in pigs. British Journal of Nutrition 50, 685-690.

Borel, P., Lairon, D., Senft, M., Chautan, M. \& Lafont, H. (1989). Wheat bran and wheat germ: effect on digestion and intestinal absorption of dietary lipids in the rat. American Journal of Clinical Nutrition 49, 1192-1202.

Bossetti, B. M., Kocher, L. M., Moranz, J. F. \& Falko, J. M. (1984). The effects of physiologic amounts of simple sugars on lipoprotein, glucose and insulin levels in normal subjects. Diabetes Care 7, 309-312.

Brillouet, J. M. \& Mercier, C. (1981). Fractionation of wheat bran carbohydrates. Journal of the Science of Food and Agriculture 32, 243-251.

Buccolo, G. \& David, H. (1973). Quantitative determination of serum triglycerides by the use of enzymes. Clinical Chemistry 19, 476-482.

Cara, L., Dubois, C., Borel, P., Armand, M., Senft, M., Portugal, H., Pauli, A. M., Bernard, P. M. \& Lairon, D. (1992). Effects of oat bran, rice bran, wheat fiber, and wheat germ on postprandial lipemia in healthy adults. American Journal of Clinical Nutrition 55, 81-88.

Cherbut, C. (1995). Effects of short chain fatty acids on gastrointestinal motility. In Physiological and Clinical Aspects of Short Chain Fatty Acids, pp. 191-207 [J. H. Cummings, J. L. Rombeau and T. Sakata, editors]. Cambridge: Cambridge University Press.

Cherbut, C., Bruley des Varannes, S., Schnee, M., Rival, M., Galmiche, J. P. \& Delort-Laval, J. (1994). Involvement of small intestinal motility in blood glucose response to dietary fibre in man. British Journal of Nutrition 71, 675-685. 
Cherbut, C. \& Ruckebusch, Y. (1985). The effect of indigestible particles on digestive transit time and colonic motility in dogs and pigs. British Journal of Nutrition 53, 549-557.

Cherbut, C., Salvador, V., Barry, J. L., Doulay, F. \& Delort-Laval, J. (1991). Dietary fibre effects on intestinal transit in man: involvement of their physicochemical and fermentative properties. Food Hydrocolloids 5,15 22.

Cummings, J. H. (1986). The effect of dietary fiber on fecal weight and composition. In Handbook of Dietary Fiber in Human Nutrition, pp. 211-280 [G. A. Spiller, editor]. Boca Raton: CRC Press.

Cummings, J. H. \& Wiggins, H. S. (1976). Transit through the gut measured by analysis of a single stool. Gut 17, $219-223$.

Dubois, C., Cara, L., Armand, M., Borel, P., Senft, M., Portugal, H., Pauli, A. M., Bernard, P.M., Lafont, H. \& Lairon, D. (1993). Effects of pea and soybean fibre on postprandial lipaemia and lipoproteins in healthy adults. European Journal of Clinical Nutrition 47, 508-520.

Edwards, C. A. (1995). Dietary fibre, fermentation and the colon. In Dietary Fibre: Mechanisms of Action in Human Physiology and Metabolism, pp. 51-60 [C. Cherbut, J. L. Barry, D. Lairon and M. Durand, editors]. Paris: John Libbey Eurotext.

Englyst, H. N., Kingman, S. M. \& Cummings, J. H. (1992). Classification and measurement of nutritionally important starch fractions. European Journal of Clinical Nutrition 46, S33-S50.

Glore, S. R., Van Treek, D., Knehans, A. W. \& Guild, M. (1994). Soluble fiber and serum lipids: a literature review. Journal of the American Dietetic Association 94, 425-436.

Grider, J. R. \& Jin, J. G. (1994). Distinct populations of sensory neurons mediate the peristaltic reflex elicited by muscle stretch and mucosal stimulation. Journal of Neurosciences 14, 2854-2860.

Guillon, F., Renard, M. G. C. C., Hospers, J., Thibault, J. F. \& Barry, J. L. (1995). Characterisation of residual fibres from fermentation of pea and apple fibres by human faecal bacteria. Journal of the Science of Food and Agriculture 68, 521-529.

Gurr, M. I. \& Asp, N. G. (1994). Dietary Fibre. ILSI Europe Concise Monograph Series. Washington: ILSI Press.

Hoebler, C., Barry, J. L., David, A. \& Delort-Laval, J. (1989). Rapid acid hydrolysis of plant cell wall polysaccharides and simplified quantitative determination of their neutral monosaccharides by gas-liquid chromatography. Journal of Agricultural and Food Chemistry 37, 360-367

Hyden, S. (1955). A turbidimetric method for the determination of higher polyethylene glycols in biological materials. Kungl. Lantbrukshögskolans Annaler (Annual Records of the Agricultural College) 22, $139-145$.

Jenkins, D. J. A., Reynolds, D., Leeds, A. R., Waller, A. L. \& Cummings, J. H. (1979). Hypocholesterolemic action of dietary fiber unrelated to fecal bulking effects. American Journal of Clinical Nutrition 32, 24302435.

Jenkins, D. J. A., Wolever, T. M. S., Rao, A. V., Hegele, R. A., Mitchell, S., Ransom, T., Boctor, D., Spadafora, P. J., Mehling, C., Katzmann Relle, L., Connelly, P. W., Story, J. A., Furamoto, E. J., Corey, P. \& Würsch, P. (1993). Effect on serum lipids of very high fiber intakes in diets low in saturated fat and cholesterol. New England Journal of Medicine 329, 21-26.

Jouany, J. P. (1982). Volatile fatty acid and alcohol determination in digestive contents, silage juices, bacterial cultures and anaerobic fermentor contents. Science des Aliments 2, 131-144.

Kashtan, H., Stern, H. S., Jenkins, D. J. A., Jenkins, A. L., Hay, K., Marcon, N., Minkin, S. \& Bruce, W. R. (1992). Wheat bran and oat bran supplements effects on blood lipids and lipoproteins. American Journal of Clinical Nutrition 55, 976-980.

Kestin, M., Moss, R., Clifton, P. M. \& Nestel, P. J. (1990). Comparative effects of three cereal brans on plasma lipids, blood pressure, and glucose metabolism in mildly hypercholesterolemic men. American Journal of Clinical Nutrition 52, 661-666.

Kuniak, L. \& Marchessault, R. H. (1972). Study of the crosslinking reaction between epichlorhydrin and starch. Die Stärke 4, 110-116.

Lampe, J. W., Slavin, J. L., Baglien, K. S., Thompson, W. O., Duane, W. C. \& Zavoral, J. H. (1991). Serum lipid and fecal bile acid changes with cereal, vegetable, and sugar beet fiber feeding. American Journal of Clinical Nutrition 52, 1235-1241.

McBurney, M. L., Horvath, P. J., Jeraci, J. L. \& Van Soest, P. J. (1985). Effect of in vitro fermentation using human faecal inoculum on the water-holding capacity of dietary fibre. British Journal of Nutrition 53, 17-24.

McConnell, A. A., Eastwood, M. A. \& Mitchell, W. D. (1974). Physical characteristics of vegetable foodstuffs that could influence bowel function. Journal of the Science of Food and Agriculture 25, 1457-1464.

Macdonald, I. (1964). The influence of dietary carbohydrates on the lipid pattern in serum and in adipose tissue. Clinical Science 27, 23-30.

Macfarlane, G. T. \& Cummings, J. H. (1991). The colonic flora, fermentation, and large bowel digestive function. In The Large Intestine: Physiology, Pathophysiology, and Disease, pp. 51-91 [S. F. Phillips, J. H. Pemberton and R. G. Shorter, editors]. New York: Raven Press.

Morgan, L. M., Tredger, J. A., Shavila, Y., Travis, J. S. \& Wright, J. (1993). The effect of non-starch polysaccharide supplementation on circulating bile acids, hormones and metabolite levels following a fat meal in human subjects. British Journal of Nutrition 70, 491-501. 
Mortensen, P. B. \& Nordgaard-Andersen, I. (1993). The dependence of the in vitro fermentation of dietary fibre to short chain fatty acids on the contents of soluble nonstarch polysaccharides. Scandinavian Journal of Gastroenterology 28, 418-422.

Narducci, F., Bassotti, G., Gaburri, M. \& Morelli, A. (1987). 24 hour manometric recording of colonic motor activity in healthy man. Gut 28, 17-25.

Prosky, L., Asp, N. G., Schweizer, T. F., De Vries, J. W. \& Furda, I. (1988). Determination of insoluble, soluble, and total dietary fiber in foods and food products: interlaboratory study. Journal of the Association of Official Analytical Chemists 71, 1017-1023.

Renaud, S., Godsey, F., Ortchanian, E. \& Baudier, F. (1979). Table de Composition des Aliments (Food Composition Table). Asnières: Astra Calvé.

Salvador, V., Cherbut, C., Barry, J. L., Bertrand, D., Bonnet, C. \& Delort-Laval, J. (1993). Sugar composition of dietary fibre and short-chain fatty acid production during in vitro fermentation by human bacteria. British Journal of Nutrition 70, 189-197.

Shinnick, F. L., Hess, R. L., Fischer, M. H. \& Marlett, J. A. (1989). Apparent nutrient absorption and upper gastrointestinal transit with fiber-containing enteral feedings. American Journal of Clinical Nutrition 49, 471475.

Siedel, J., Hägele, E. O., Ziegenhorn, J. \& Wahlefeld, A. W. (1983). Reagent for the enzymatic determination of serum total cholesterol with improved lipolytic efficiency. Clinical Chemistry 29, 1075-1080.

Sievet-Desrumeaux, C., Dedomder-Decoopman, E., Fruchart, J. C., Dewailli, P. \& Sezille, G. (1979). Immunochemical determination of human apolipoprotein B by laser nephelometry. Clinica Chimica Acta 95, 405-408.

Sievet-Desrumeaux, C., Dedomder-Decoopman, E., Fruchart, J. C., Dewailli, P., Sezille, G. \& Jaillard, J. (1980). Immunochemical determination of human apolipoprotein A-1 by laser nephelometry. Clinica Chimica Acta 107, 145-148.

Spiller, G. A. (1986), Suggestions for a basis on which to determine a desirable intake of dietary fiber. In Handbook of Dietary Fiber in Human Nutrition, pp. 281-283 [G. A. Spiller, editor.] Boca Raton: CRC Press.

Stephen, A. (1994). Propionate - sources and effect on lipid metabolism. In Short Chain Fatty Acids. Falk Symposium no. 73, pp. 260-271 [H. J. Binder, J. H. Cummings and K. H. Soergel, editors]. Lancaster: MTP Press Limited.

Stevens, B. J. H. \& Selvendran, R. R. (1988). Changes in composition and structure of wheat bran resulting from the action of human faecal bacteria in vitro. Carbohydrate Research 183, 311-319.

Stevens, B. J. H., Selvendran, R. R., Bayliss, C. E. \& Turner, R. (1988). Degradation of cell wall material of apple and wheat bran by human faecal bacteria in vitro. Journal of the Science of Food and Agriculture 44, $151-166$

Story, J. A. \& Kritchevsky, D. (1976). Comparison of the binding of various bile acids and bile salts by several types of fiber. Journal of Nutrition 106, 1292-1294.

Takayama, M., Itoh, S., Nagasaki, T. \& Tanimizu, I. (1977). A new enzymatic method for choline containing phospholipids. Clinica Chimica Acta 79, 93-98.

Thibault, J. F. (1979). Automatisation du dosage des substances pectiques par la méthode au métahydroxydiphényl (An automatised method for the determination of pectic substances). LebensmittelWissenschaft und Technologie 12, 247-251.

Van Soest, P., Horvath, P., McBurney, M., Jeraci, J. \& Allen, M. (1983). Some in vitro and in vivo properties of dietary fibers from noncereal sources. In Unconventional Sources of Dietary Fiber. Symposium Series no. 214, pp. 135-141 [I. Furda, editor]. Washington, DC: American Chemical Society.

Wolever, T. M. S. (1995). Dietary fibre and lipid metabolism in humans. In Dietary Fibre: Mechanisms of Action in Human Physiology and Metabolism, pp. $69-81$ [C. Cherbut, J. L. Barry, D. Lairon and M. Durand, editors]. Paris: John Libbey Eurotext.

Wolever, T. M. S., Jenkins, D. J. A., Mueller, S., Boctor, D. L., Ransom, T. P. P., Patten, R., Chao, E. S. M., McMillan, K. \& Fulgoni, V. (1994). Method of administration influences the serum cholesterol-lowering effect of psyllium. American Journal of Clinical Nutrition 59, 1055-1059. 